\title{
Integrated planning of linear infrastructure and conservation offsets
}

\author{
J. D. Bunton ${ }^{\text {a }}$, A. T. Ernst ${ }^{\text {a, }}$ J. O. Hanson ${ }^{\text {b }}$, H. L. Beyer ${ }^{\text {b }}$, E. Hammill ${ }^{\text {b }}$, C. A. Runge ${ }^{\text {b }}$, O. Venter ${ }^{\text {b }}$, \\ H. P. Possingham ${ }^{b}$ and J. R. Rhodes ${ }^{b}$ \\ ${ }^{a}$ CSIRO Digital Productivity Flagship, Gate 5 Normanby Road, Clayton VIC, 3168 \\ ${ }^{\mathrm{b}}$ ARC Centre of Excellence for Environmental Decisions, Centre for Biodiversity \& Conservation Science, \\ University of Queensland, Brisbane QLD 4072, Australia \\ Email: Joe.Bunton@csiro.au
}

\begin{abstract}
Linear infrastructure (e.g. roads, railways, utility corridors) is critical to the functioning of modern industrialised societies, but has profound impacts on ecological systems and their biological diversity. The ecological impacts of linear infrastructure may be exacerbated by the failure to anticipate and plan for them early on. Moreover, poor planning can lead to the costly redesign or abandonment of projects to avert unforeseen ecological impacts. Explicitly considering trade-offs between linear infrastructure development and conservation requirements (see Figure 1) at an early planning stage provides the greatest opportunities for achieving both routing and offsetting objectives cost-effectively.
\end{abstract}

Here, we combine route planning for linear infrastructure with strategic conservation offset decision making in a unified framework to identify efficient opportunities for both routing and impact mitigation. The routing component is spatially explicit and links two or more target nodes via a network of connected planning units with fixed costs while accounting for impacts on features of conservation concern. The offsetting component identifies efficient solutions for mitigating the unavoidable impacts of linear infrastructure. We demonstrate the benefits of solving both planning objectives simultaneously by contrasting the cost-effectiveness of the integrated solutions with those found by solving the problems sequentially.
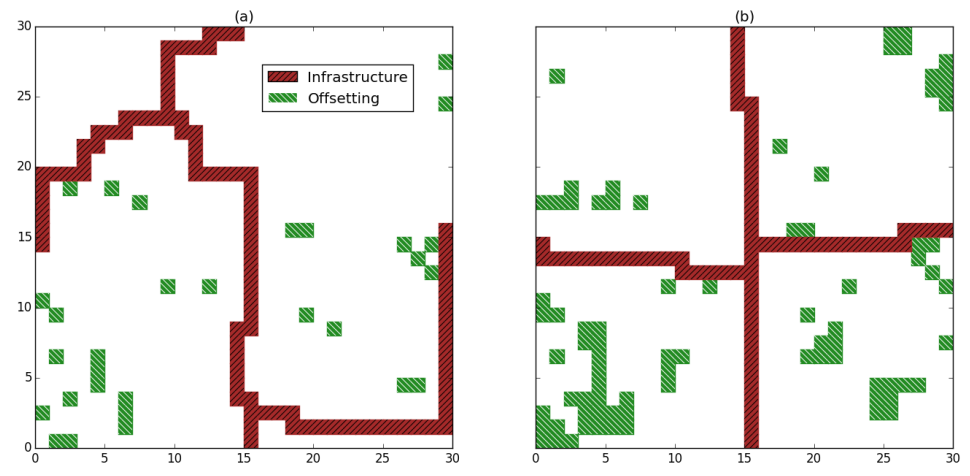

Figure 1. Solutions to a problem instance, favouring (a) offsetting and (b) infrastructure.

We formulated these planning problems in a mathematical optimisation framework in which space is discretised into planning units. Four sets of attributes were quantified for each planning unit as inputs to the optimisation problem: (i) the expected loss of conservation value resulting from linear infrastructure development for each of the conservation features (e.g. species); (ii) the cost of linear infrastructure development; (iii) the cost of offsetting; and (iv) the estimated benefit of offsetting with respect to the conservation features. The decision variables determine whether each planning unit is part of the routing solution, the offsetting solution, or neither. The mitigation effort is required to be sufficient to offset all of the impacts on biodiversity (no net loss) generated by the linear infrastructure development.

We describe an integer linear programming formulation of the optimisation problem and evaluate its efficacy through simulation. We find that solving both problems simultaneously can dramatically improve efficiency compared to solving the routing and offsetting problems sequentially. Furthermore, a sequential approach resulted in infeasible offsetting problems in $25 \%$ of cases. This work suggests both linear infrastructure and conservation offset planning could benefit from greater coordination in the early stages of the planning process.

Keywords: Conservation planning, linear infrastructure, mixed integer programming, optimisation, offsetting 
Bunton, Ernst, Hanson et al. Integrated planning of linear infrastructure and conservation offsets

\section{INTRODUCTION}

The global economy depends upon efficient and effective transport infrastructure (Rodrigue et al., 2013), including roads, railways, and pipelines. Maintenance of future economic growth will require construction of new transport infrastructure to meet demand. For example, the construction of more than 25 million kilometres of new roads is anticipated globally by 2050, representing a 60\% increase over 2010 levels (Laurance et al., 2014). This unprecedented level of construction has the potential to substantially impact global biodiversity, especially considering the majority of construction is predicted to occur in biodiverse developing nations (Dulac, 2013). The predicted increase in construction of transportation infrastructure, and its potential to detrimentally impact the global environment necessitates the development of strategic planning tools that are able to meet industry demands while minimising environmental impacts. However, existing approaches (e.g. impact assessment, offsetting) focus predominantly on costs, impacts, or ah hoc mitigation, and lack a strategic planning focus.

The direct environmental impacts of linear infrastructure (LI) include loss and fragmentation of habitat, mortality from collisions, disruption of migration routes, chemical pollution and dust, behavioural disturbance, and alterations to the physical environment (Forman and Alexander, 1998; Fahrig and Rytwinski, 2009; Kociolek et al., 2011). Indirect effects include changes to population distribution and abundance, edge effects, invasion by non-native species, further anthropogenic development, and altered species interactions (Fahrig et al., 1995; Saunders et al., 1991; Trombulak and Frissell, 2000). Mitigating such a diverse array of ecological impacts is difficult. Approaches to reducing impacts include strategic routing of LI, providing crossing structures for wildlife (overpasses or underpasses), fencing, imposing speed restrictions in high risk areas, and closing routes during key migration periods. These strategies can be effective at mitigating a subset of impacts arising from linear infrastructure development (Forman et al., 2012).

Many impacts cannot be mitigated, however, and offsetting - protecting or improving habitat in other areas to compensate for these unavoidable impacts - is now widely employed as a framework for balancing development with environmental protection such that, at a minimum, there should be no net loss (NNL) of biodiversity or ecosystem function overall (McKenney and Kiesecker, 2010; Gordon et al., 2011). NNL requires quantification of the losses resulting from development and the benefits resulting from offsetting relative to a baseline describing the expected change in biodiversity in the absence of development (Gordon et al., 2015). Importantly, if the baseline used to quantify benefit is itself a decline in biodiversity over time then biodiversity loss may still result, but not as fast as would have occurred had the development not taken place (Maron et al., 2013). Quantifying a baseline that appropriately captures all dimensions of impact is difficult (Moreno-Mateos et al., 2015) and evidence suggests offsetting may not achieve its stated aim of NNL in practice (Curran et al., 2014; Gordon et al., 2015; Maron et al., 2015). Nevertheless, offsetting is common and can be a major cost in many development projects.

Given that there can be flexibility in LI routing and offsetting options, there is potential to reduce environmental losses and the risk of resulting project delays and design amendments by explicitly considering environmental impacts and offsetting options when planning for LI. In addition, simultaneously carrying out landscape planning and strategic offsetting has been shown to achieve substantial benefits in terms of cost reduction and number of species conserved (Underwood, 2011). Close collaboration between LI design and conservation offsets can be facilitated by providing tools that allow near real-time evaluation of trade-offs between infrastructure and environmental objectives under routing and offset options.

Here, we quantify the efficiency gains that are made possible by combining LI routing and offset selection in the early stages of planning of a development project to address the direct, unavoidable impacts of development on biodiversity. We assume that an appropriate baseline or counterfactual has been identified to quantify both impacts of development and benefits of offsetting (Bull et al., 2014; Maron et al., 2015) and address how this information can then be used to improve planning. Solving both problems simultaneously, which has not been attempted previously, provides opportunities to benefit from changes to LI routing to avoid areas of high biodiversity value that would be costly, difficult or impossible to offset or that could threaten the viability of the project. Where areas of biodiversity are not avoidable, optimal offsetting strategies can be determined.

\subsection{Network Design}

The hypothetical LI problem considered in this paper is to connect a given set of points with a minimum cost set of intermediate areas. As presented here the cost of building LI is assumed to depend only on the cost of planning areas through which it passes, not on the direction. However including direction-dependent costs 
would be trivial in our formulation.

Since we are dealing with a graph induced by the discrete planning units and their adjacencies, the LI routing problem can be considered a subclass of Network Design Problems (NDP). NDP can be formulated generally (Feremans et al., 2003) or more specifically to take advantage of problem characteristics.

The cost of building LI means that the optimal solution will not include any additional links beyond those required to connect the terminals. Hence this is a type of Steiner Tree Problem. The Steiner tree problem involves finding the minimum distance network or subgraph that links $T$ points (known as terminal nodes) in the Euclidean Plane or on a graph. Intermediate nodes of the connecting network are called steiner nodes.

The original motivation for our problem is continuous and could be approached using continuous models (Brazil and Zachariasen, 2015; Demaine et al., 2013). However, based on the discretisation used for the environmental assessment we have a graph. Steiner tree problems on graphs are NP-complete (no quick solution method is known, however solutions can be verified quickly) (Hwang and Richards, 1992). The best known worst case performance for Steiner tree problems on graphs is given by Hougardy et al. (2014), using a dynamic programming approach. Specialised methods, based for example on branch-and-cut, can solve large instances with many hundreds of nodes and thousands of edges (see (Gamrath et al., 2014; Pajor et al., 2014)).

\subsection{The Combined Problem}

To quantify the efficiency gains obtainable by using a combined approach to LI routing and offsetting, we compare it to the cost of first minimising the LI costs and then the offset costs. That is, we can treat it as a multi-criteria problem or as a hierarchical optimisation problem. For multi-criteria problems there can be multiple pareto-optimal solutions that provide a different trade-off between the two objectives. In our experiments we do not attempt to map out the complete pareto frontier but create solutions using pre-specified relative weights of the objectives.

Hierarchical optimisation involves the treatment of different criteria one at a time. During the first phase offset requirements are ignored. In the second phase the linear infrastructure decisions are fixed and only offsets optimised $^{1}$. The two phase approach results in simpler problems to solve as only decisions relating to the relevant criteria need to be considered. Due to this, solutions achieved are not guaranteed to be globally optimal. This approach mirrors the common practice in LI routing of completing the route planning before commencement of conservation offsetting considerations.

\section{Problem Formulation}

Here we formulate the joint LI routing and offsetting problem as a Mixed-Integer Programming (MIP) problem. The offsetting problem is formulated as a site selection problem similar to the conservation planning software MARXAN (Ball et al., 2009; Watts et al., 2009). The LI routing problem is formulated as a disaggregated Fixed Charge Network Flow problem (FCNF) with no flow costs following the standard practice for steiner tree problems (Goemans and Myung, 1993). Linking the two separate formulations are two sets of constraints: firstly, that each planning unit may may not be both LI and an offsetting zone; secondly, that the offsetting target for the reserve selection problem is the amount of damage to features done by the planning units chosen to be LI.

Using the notation described in Table 1, we can formulate the problem as an mixed-integer linear program. Note that we are only using the minimum number of pairs of terminals to ensure a connected graph.

\footnotetext{
${ }^{1}$ A slightly more general bi-criterion method is to bound the cost of the linear infrastructure in the second phase to the minimum achieved in the first phase. For non-uniform costs where the minimum linear infrastructure solution is unique this is expected to be equivalent.
} 
Bunton, Ernst, Hanson et al. Integrated planning of linear infrastructure and conservation offsets

Table 1. Sets, Variables and Parameters for Formulation

\begin{tabular}{ccl} 
Component & Type & Description \\
\hline \hline$I$ & Set & Set of Planning Units $i \in I$ \\
$N_{i}$ & Set & Set of Planning Units $j \in N_{i}$ that neighbours with Planning Units $i \in I$ \\
$F$ & Set & Set of Features $f \in F$ \\
$T$ & Set & Set of terminals $i \in T$ that are to be connected by LI \\
$P$ & Set & Pairs of flows equivalent to connecting all locations $p \in P$ \\
\hline$X_{i}$ & Variable & If Planning Unit $i \in I$ is allocated to be a offsetting zone \\
$Y_{i}$ & Variable & If Planning Unit $i \in I$ is allocated to be LI \\
$Z_{i, j}$ & Variable & Indicator if both Planning Units $i \in I$ and $j \in N_{i}$ are offsetting zones \\
$F l_{i, j, p}$ & Variable & Flow from Planning unit $i \in I$ to $j \in N_{i}$ for flow $p \in P$ \\
\hline$R_{i, f}^{o}$ & Parameter & Value for occurrence of feature $f \in F$ for Planning Unit $i \in I$ \\
$R_{i, f}^{r}$ & Parameter & Value for restoration of feature $f \in F$ for Planning Unit $i \in I$ \\
$C_{i}^{L I}$ & Parameter & Cost for allocating Planning Unit $i \in I$ as LI \\
$C_{i}^{o f f s e t}$ & Parameter & Cost for allocating Planning Unit $i \in I$ for offsetting \\
$b$ & Parameter & Value for having a boundary between two offsetting zones. As this is a \\
& & bonus, $b<0$ is a negative cost \\
$m$ & Parameter & a constant multiplier that can be used to force the model to achieve greater \\
& & offsetting benefits than losses (if $\mathrm{m}>1$ ) to compensate for underestimates \\
& in impacts & \\
$v_{i, j}$ & Parameter & Value of boundary (i.e. boundary length) between Planning unit $i \in I$ \\
& & and $j \in I$ \\
$s o_{i, p}$ & Parameter & 1 if Planning Unit $i \in I$ is the source for pair $p \in P$ \\
$s i_{i, p}$ & Parameter & 1 if Planning Unit $i \in I$ is the sink for pair $p \in P$ \\
\hline
\end{tabular}

Objective: $\quad \min \sum_{i \in I} \quad\left(C_{i}^{\text {offset }} X_{i}+C_{i}^{L I} Y_{i}+b \sum_{j \in N_{i}} v_{i, j} Z_{i, j}\right)$

Flow conservation:

$\sum_{j \in N_{i}} F l_{i, j, p}+s i_{i, p}=\sum_{j \in N_{i}} F l_{j, i, p}+s o_{i, p} \forall i \in I, p \in P$

Only allow flow if LI is built:

$\sum_{j \in N_{i}} F l_{j, i, p} \leq Y_{i}$

$\forall i \in I, p \in P$

Fix terminals to be LI:

$Y_{i}=1$

$\forall i \in T$

Can't be both LI and an offsetting zone: $X_{i}+Y_{i} \leq 1$

$\forall i \in I$

NNL for features damaged by LI: $\quad \sum_{i \in I} R_{i, f}^{r} X_{i} \geq m \sum_{i \in I} R_{i, f}^{o} Y_{i}$

$\forall f \in F$

Linearisation of boundary variables:

$$
Z_{i, j} \leq X_{i} \quad \text { and } \quad Z_{i, j} \leq X_{j}
$$

$\forall i \in I, \quad j \in N_{i}$
Binary Variables:
$X_{i}, Y_{i} \in\{0,1\}$
$\forall i \in I$
Continuous Variables:
$0 \leq Z_{i, j}, F l_{i, j, p} \leq 1$
$\forall i \in I, j \in N_{i}, p \in P$

Equation (1) defines the objective as the sum of the costs of offsetting zones and LI, and the sum of boundary benefits from adjacent offsetting zones. Equations (2), (3) \& (4) define the Flows for the LI routing problem, ensuring flow: is conserved, requires LI to be built, and has terminals as the sources and sinks. Equations (5) \& (6) link the LI routing and offsetting problems, ensuring planning units cannot be both LI and offsetting zones, and that the offsetting target equals the damage to features from LI. Equation (7) linearises the boundary variables, ensuring the boundary benefit is only given to neighbouring offsetting zones.

\section{Computational Study}

All tests were performed running the commercial solver software CPLEX 12.5.0.1 on quad octa core 1.87GHz Xeon L7555 processors and 64GB of RAM running SUSE Linux Enterprise Server 11. Each run was 
done using 8 cores for 1 hour real time, or until 1.00\% MIP gap was reached. The MIP gap is defined as (ObjectiveValue - ObjectiveBound)/|ObjectiveValue| for a minimisation problem such as ours, with a MIP gap of 0 meaning we have an optimal solution.

Each run was specified by a number of parameters: $p u$ - size of planning area, a square of $p u \times p u$ planning units; $s p$ - the number of features being considered (i.e. species); $r d$ - the number of LI nodes to be linked, these are evenly distributed around the edges of the planning area; dam - scaling of LI damage; ratio - the ratio of scaling factors offsetting zone to LI cost (ratio $>1$ means the offsetting cost is scaled higher than LI cost), which was used to investigate how the relative cost of development and offsetting influenced the solutions. The offset requirement scaling $(m)$ was set to 1 for all problems. Problems instances were solved using both the combined (1 phase) and hierarchical (2 phase) approaches.

For a given set of parameters, planning units were generated and assigned costs for LI and for offset actions. To ensure that the simulated species' distributions were spatially autocorrelated and limited in range size, the presence/absence of each species in the planning units were simulated according to a random Bernoulli process (using a Gaussian covariance model with a scale parameter of 3 and a threshold parameter of 0.3) (Schlather et al., 2015).

Table 2. Mean and standard deviation of MIP gap for 1 phase (combined) problems, and Mean Solve Times (MST) for 1 phase and 2 phase approaches, grouped by different problem characteristics.

\begin{tabular}{llcccc} 
& Characteristic & Mean MIP Gap (\%) & MIP Gap SD (\%) & 1 Phase MST (s) & 2 Phase MST (s) \\
\hline \hline$p u=$ & 15 & 4.23 & 4.93 & 1460 & 2402 \\
& 20 & 11.34 & 10.56 & 2413 & 2033 \\
& 30 & 21.25 & 13.45 & 3131 & 3185 \\
\hline$s p=$ & 3 & 9.68 & 10.85 & 1900 & 2419 \\
& 10 & 16.52 & 13.54 & 2771 & 2740 \\
\hline$r d=$ & 4 & 2.18 & 3.39 & 1027 & 885 \\
& 6 & 16.49 & 11.15 & 3030 & 3607 \\
\hline dam $=$ & 1 & 21.59 & 12.15 & 2971 & 3605 \\
\hline ratio $=$ & 0.01 & 12.78 & 11.92 & 2473 & 2559 \\
& 0.1 & 12.87 & 13.41 & 2203 & 2596 \\
\hline 1 & 13.34 & 11.71 & 2581 & 2571 \\
10 & 13.70 & 11.68 & 2633 & 2553 \\
100 & 13.54 & 11.98 & 2658 & 2613 \\
& 1000 & 13.54 & 13.38 & 2283 & 2577 \\
& 11.28 & 12.67 & 1973 & 2536
\end{tabular}

Table 2 presents the results of our runs, grouping all runs done by different problem characteristics to observe their effects on performance. Most of the complexity of the problem comes from the LI routing Steiner Tree Problem, as shown by the similar mean solve times for the 1 phase vs 2 phase approaches. Problem difficulty is most sensitive to $p u$ and $r d$. This is intuitive as the parameters increase the difficulty of the more challenging LI routing aspect of the problem, introducing additional constraints and variables due to adding planning units and flows to the problem respectively. Problem difficulty also increases with $s p$, which increases the difficulty of the offsetting component of the problem. The parameters dam and ratio appear to have had less of an effect on performance.

Figure 2 compares the percentage of problem instances solved under specified MIP Gaps for the 1 phase and 2 phase problems. As the bounds generated in the 2 phase approach are not for the combined problem, the MIP gaps for the 2 phase problems were calculated using the best bounds from the 1 phase approach. There is little difference for the best solved 35\% of problems, however better MIP gaps are consistently reached by the 1 phase approach for the rest of the problems. In fact, the 1 phase approach achieves solutions to all feasible problems, whereas the 2 phase approach fails to find a feasible solution for $25 \%$ of problems. This $25 \%$ failure rate occurs as the LI routing solution initially achieved in the 2 phase approach generates a landscape where offsetting the ecological damage is impossible. This is akin to the real world situation where a proposed LI route is unable to be satisfactorily offset, effectively requiring a complete revision of routing. 


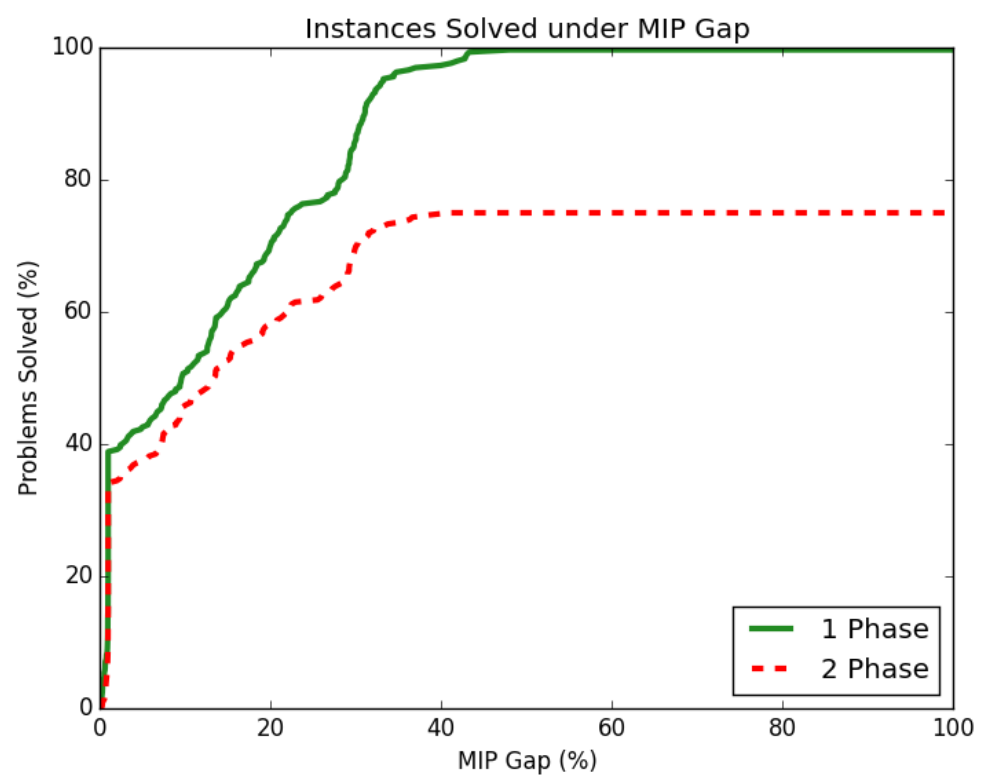

Figure 2. Instances solved below given MIP gap for multi-criteria \& hierarchical approaches.

\section{Conclusions And Future Directions}

The benefits of the combined approach to LI routing and conservation offsetting include more efficient solutions, lower overall conservation impacts, and increased probability of finding a feasible solution. Specifically, the hierarchical approach generated LI routes that resulted in infeasible conservation offsetting problems in $25 \%$ of instances, while the combined approach was universally able to achieve feasible solutions. Although offsetting sites could be selected from a wider area in the real world to achieve a specified target, these results do illustrate the potential benefits of considering both problems simultaneously.

The little difference in mean solve times between 1 phase and 2 phase approaches indicates a small increase in problem complexity by considering offsetting and biodiversity aspects on top of LI planning considerations. This is encouraging as it suggests a low barrier for entry for integrated planning.

While problem difficulty scales quickly with problem size, we are able to solve many reasonably sized problems to near optimality. This may be improved upon with further performance considerations.

Though we have demonstrated several potential benefits of a combined approach to LI routing, this framework would benefit from considering non-localised offsetting, as well as the inclusion of a more realistic treatment of the biodiversity damage calculations, offsetting options, and LI routing constraints.

There is considerable interest in moving towards more strategic landscape-level planning for biodiversity conservation and we have presented a novel approach for achieving this. Both conservation and LI routing objectives can benefit from greater coordination in the early stages of the development process. In many situations the increased costs associated with this planning coordination are likely to be small relative to the potential offsetting cost savings and reduced risk of catastrophic planning failure with respect to environmental impacts. Furthermore, demonstrating that environmental considerations have been formally and quantitatively integrated into the planning process is likely to make planning proposals or contract bids more competitive.

\section{REFERENCES}

Ball, I. R., H. Possingham, and M. E. Watts (2009, May). Marxan and relatives: Software for spatial conservation prioritisation. Chapter 14. In Spatial Conservation Prioritization: Quantitative Methods and Computational Tools, pp. 185-195. Oxford University Press.

Brazil, M. and M. Zachariasen (2015). Steiner Trees with Other Cost Functions and Constraints. In Optimal Interconnection Trees in the Plane, Number 29 in Algorithms and Combinatorics, pp. 219-299. Springer International Publishing.

Bull, J. W., A. Gordon, E. A. Law, K. B. Suttle, and E. J. Milner-Gulland (2014, June). Importance of Baseline Specification in Evaluating Conservation Interventions and Achieving No Net Loss of Biodiversity. Conservation Biology 28(3), 799-809. 
Bunton, Ernst, Hanson et al. Integrated planning of linear infrastructure and conservation offsets

Curran, M., S. Hellweg, and J. Beck (2014, June). Is there any empirical support for biodiversity offset policy? Ecological Applications 24(4), 617-632.

Demaine, E. D., M. Hajiaghayi, and P. N. Klein (2013, June). Node-Weighted Steiner Tree and Group Steiner Tree in Planar Graphs. ACM Trans. Algorithms 10(3), 13:1-13:20.

Dulac, J. (2013). Global land transport infrastructure requirements. Estimating road and railway infrastructure capacity and costs to 2050. International Energy Agency.

Fahrig, L., J. H. Pedlar, S. E. Pope, P. D. Taylor, and J. F. Wegner (1995). Effect of road traffic on amphibian density. Biological Conservation 73(3), 177-182.

Fahrig, L. and T. Rytwinski (2009, June). Effects of roads on animal abundance: an empirical review and synthesis. Ecology and Society 14(1), 21.

Feremans, C., M. Labbe, and G. Laporte (2003, July). Generalized network design problems. European Journal of Operational Research 148(1), 1-13.

Forman, R. T., J. P. Beckmann, A. P. Clevenger, M. Huijser, and J. A. Hilty (2012). Safe passages: highways, wildlife, and habitat connectivity. Island Press.

Forman, R. T. T. and L. E. Alexander (1998). Roads and their major ecological effects. Annual Review of Ecology and Systematics 29, 207-231.

Gamrath, G., T. Koch, D. Rehfeldt, and Y. Shinano (2014, September). SCIP-Jack - A massively parallel STP solver.

Goemans, M. X. and Y.-S. Myung (1993, January). A catalog of steiner tree formulations. Networks 23(1), 19-28.

Gordon, A., J. W. Bull, C. Wilcox, and M. Maron (2015, April). Perverse incentives risk undermining biodiversity offset policies. Journal of Applied Ecology 52(2), 532-537.

Gordon, A., W. T. Langford, J. A. Todd, M. D. White, D. W. Mullerworth, and S. A. Bekessy (2011, December). Assessing the impacts of biodiversity offset policies. Environmental Modelling \& Software 26(12), 1481-1488.

Hougardy, S., J. Silvanus, and J. Vygen (2014, June). Dijkstra meets Steiner: a fast exact goal-oriented Steiner tree algorithm. arXiv:1406.0492 [cs].

Hwang, F. K. and D. S. Richards (1992, January). Steiner tree problems. Networks 22(1), 55-89.

Kociolek, A. V., A. P. Clevenger, C. C. St. Clair, and D. S. Proppe (2011, April). Effects of Road Networks on Bird Populations. Conservation Biology 25(2), 241-249.

Laurance, W. F., G. R. Clements, S. Sloan, C. S. O’Connell, N. D. Mueller, M. Goosem, O. Venter, D. P. Edwards, B. Phalan, A. Balmford, R. Van Der Ree, and I. B. Arrea (2014, September). A global strategy for road building. Nature 513(7517), 229-232.

Maron, M., J. W. Bull, M. C. Evans, and A. Gordon (2015). Locking in loss: Baselines of decline in Australian biodiversity offset policies. Biological Conservation. DOI: 10.1016/j.biocon.2015.05.017.

Maron, M., J. R. Rhodes, and P. Gibbons (2013, September). Calculating the benefit of conservation actions. Conservation Letters 6(5), 359-367.

McKenney, B. A. and J. M. Kiesecker (2010, January). Policy development for biodiversity offsets: a review of offset frameworks. Environmental Management 45(1), 165-176.

Moreno-Mateos, D., V. Maris, A. BÃl'chet, and M. Curran (2015). The true loss caused by biodiversity offsets. Biological Conservation. DOI: 10.1016/j.biocon.2015.08.016.

Pajor, T., E. Uchoa, and R. F. Werneck (2014). A Robust and Scalable Algorithm for the Steiner Problem in Graphs. In Presented at the 11th DIMACS Implementation Challenge on Steiner Tree Problems, arXiv:1412.2787 [cs], pp. 1-33.

Rodrigue, J.-P., C. Comtois, and B. Slack (2013). The geography of transport systems. Routledge.

Saunders, D. A., R. J. Hobbs, and C. R. Margules (1991, March). Biological consequences of ecosystem fragmentation - a review. Conservation Biology 5(1), 18-32.

Schlather, M., A. Malinowski, P. J. Menck, M. Oesting, and K. Strokorb (2015). Analysis, simulation and prediction of multivariate random fields with package RandomFields. Journal of Statistical Software 63(8), $1-25$.

Trombulak, S. C. and C. A. Frissell (2000, February). Review of ecological effects of roads on terrestrial and aquatic communities. Conservation Biology 14(1), 18-30.

Underwood, J. G. (2011, January). Combining landscape-level conservation planning and biodiversity offset programs: a case study. Environmental Management 47(1), 121-129.

Watts, M. E., I. R. Ball, R. S. Stewart, C. J. Klein, K. Wilson, C. Steinback, R. Lourival, L. Kircher, and H. P. Possingham (2009, December). Marxan with Zones: Software for optimal conservation based land- and sea-use zoning. Environmental Modelling \& Software 24(12), 1513-1521. 